\title{
Análisis de falla de encendido en motores de combustión utilizando señales de vibración basado en el cálculo y reducción de indicadores estadísticos
}

\author{
Misfire Analysis in Combustion Engines Using Vibration Signals Based on the Calculation and \\ Reduction of Statistical Indicators
}

\section{Alejandro García-Basurto ${ }^{1}$, Juan José Saucedo-Dorantes², Ángel Pérez-Cruz³ ${ }^{3}$ Roque Alfredo Osornio-Ríos ${ }^{4}$}

\author{
Universidad Autónoma de Querétaro, MÉxICO \\ 1alejandro.garcia.basurto@uaq.mx \\ 2https://orcid.org/0000-0001-9026-6694 | juan.saucedo@uaq.mx \\ 3https://orcid.org/0000-0001-5320-4064 | angel.perez@uaq.mx \\ 4https://orcid.org/0000-0003-0868-2918 | raor@uaq.mx
}

Recibido 12-10-2020, aceptado 08-12-2020.

\begin{abstract}
Resumen
En este trabajo de investigación se propone una metodología de diagnóstico basada en el cálculo y reducción de indicadores estadísticos estimados de señales de vibración para la detección de fallas de encendido o por chispa en un motor de combustión interna (MCI). El trabajo realizado por los autores incluye la caracterización de señales de vibración mediante cuatro indicadores estadísticos capaces de modelar la tendencia y describir cambios en las señales. Además, en el método propuesto se introduce el uso de las técnicas de reducción de indicadores estadísticos como análisis de componentes principales y análisis discriminante lineal las cuales tienen la capacidad de reducir la dimensionalidad de la base del conjunto original de indicadores en el dominio del tiempo y obtener representaciones visuales de los patrones característicos de las condiciones de operación evaluadas. El método propuesto se evalúa sobre datos experimentales adquiridos durante la operación normal y con falla de encendido de un MCI en régimen de velocidad baja y alta. El procesamiento de las señales de vibración adquiridas y la aplicación de la metodología de diagnóstico propuesta se hizo bajo el entorno de programación de Matlab. Los resultados obtenidos en patrones característicos bidimensionales presentan claramente la separación de las diferentes condiciones de operación, dichos resultados demuestran que la metodología permite detectar fallas en MCI generadas en el sistema de encendido y que la detección de las fallas se puede realizar de forma efectiva independientemente de la velocidad de giro del motor.
\end{abstract}

Palabras clave: Análisis discriminante lineal, análisis de vibraciones, detección de fallas, indicadores estadísticos, motor de combustión interna.

\begin{abstract}
In this research work, a diagnosis methodology based on the calculation and reduction of statistical features that are obtained from vibration signals is proposed for the detection of misfire or spark failure in an internal combustion engine (ICE). This work is carried out by the characterization of vibration signals using four statistical features that are capable of modeling trends and describing changes in the acquired vibration signals. In addition, the proposed method introduces the consideration of dimensionality reduction techniques such as principal component analysis and linear discriminant analysis, which allows to obtain the reduction of an original feature space leading to achieve a visual representation of the characteristic patterns, represented by the set of statistical features, that characterizes different evaluated conditions in the ICE. The proposed method is evaluated on experimental data acquired during normal and misfire operation of an ICE in low and high speed regime. The processing of the acquired vibration signals and the application of the proposed diagnosis methodology is performed under Matlab ${ }^{\circledR}$, which is a sophisticated software that may be used in a wide range of engineering applications. The results obtained in two-dimensional characteristic patterns clearly show the characterization of different operating conditions evaluated in the ICE, these results demonstrate that the methodology allows detecting faults in an ICE that are generated in the ignition system and that the detection of faults can be carried out effectively regardless of the rotational speed of the engine.
\end{abstract}

Index terms: Fault detection, internal combustion engine, linear discriminant analysis, statistical indicators, vibration analysis. 


\section{INTRODUCCIÓN}

En las últimas décadas, los avances y desarrollos tecnológicos han influenciado directamente sobre diferentes áreas generando nuevas tendencias. En este sentido, la industria automotriz no ha quedado exenta de dichos avances, sin embargo, en México, aunque se han presentado grandes avances que van desde la optimización del consumo de combustible hasta la aplicación de motores híbridos y eléctricos, aún en la actualidad circulan millones de automóviles impulsados por motores de combustión interna (MCI). En este sentido, existe una gran necesidad en los talleres de servicio automotriz por desarrollar nuevas herramientas aplicadas al monitoreo, diagnóstico y detección de fallas en MCI con el objetivo de incrementar su vida útil y minimizar los gastos de mantenimiento.

La problemática actual radica en que la aparición de fallas de encendido o por chispa en la bujía en MCI son las más comunes y pueden darse de forma repentina produciendo principalmente pérdidas de potencia y altas emisiones contaminantes, afectando su desempeño (véase Tabla 1) [1]. Por otra parte, el diagnóstico de fallas producidas en el sistema de encendido se realiza tradicionalmente en forma heurística, lo que implica en la mayoría de los casos, que se debe de realizar una inspección física de los componentes y en consecuencia un criterio de diagnóstico individual. Aunque existen herramientas que asisten en el diagnóstico de este tipo de fallas, generalmente el acceso a estas herramientas es drásticamente limitado por ser herramientas comercialmente costosas.

TABLA 1 SISTEMAS QUE PUEDEN CAUSAR PÉRDIDA DE POTENCIA, ALTAS EMISIONES Y BAJO DESEMPEÑO DEL MCI

\begin{tabular}{ccc}
\hline $\begin{array}{c}\text { Sistemas del Motor de } \\
\text { Combustión Interna }\end{array}$ & $\begin{array}{c}\text { Cantidad de } \\
\text { Causas }\end{array}$ & Porcentaje \\
\hline Sistema de Encendido & 9 & $30 \%$ \\
\hline Sistema de Tren Valvular & 7 & $23.3 \%$ \\
\hline $\begin{array}{c}\text { Sistema de Inyección de } \\
\text { Combustible }\end{array}$ & 6 & $20 \%$ \\
\hline Sistema de Emisiones & 5 & $16.7 \%$ \\
\hline Sistema de Distribución & 2 & $6.7 \%$ \\
\hline Otros Sistemas & 1 & $3.3 \%$ \\
\hline
\end{tabular}

Asimismo, la identificación de fallas en MCI ha representado un tema interesante para algunos investigadores que intentan resolver problemáticas que pueden presentarse durante el diagnóstico. En este sentido, tanto el monitoreo y adquisición de diferentes señales, como el uso de técnicas de procesamiento aplicadas como método de diagnóstico y detección de fallas, son una de las alternativas y enfoques que se ha estudiado en los últimos años. Así, entre las señales más utilizadas se encuentran las vibraciones y por ejemplo, mediante la representación de la señales de vibración en mapas del dominio tiempo-frecuencia estimadas por el uso de la transformada continua Wavelet (CWT, Continuous Wavelet Transform) se ha logrado demostrar que las vibraciones del MCI representan una medida fiable y apropiada para la identificación de fallas [2]. También se propuso una técnica de diagnóstico de fallas en MCI basada en patrones de puntos visuales de las señales acústicas y de vibración adquiridas, los resultados experimentales indicaron que el método propuesto es efectivo para la identificación de fallas en el MCI [3]. Además, existen otros trabajos que han logrado realizar la identificación de fallas en los sistemas de encendido, combustión, e inyección mediante el procesamiento de señales de vibración, en donde, el cálculo de indicadores estadísticos representa una estrategia aceptable para analizar la tendencia y cambios de señales [4]. De igual forma se han logrado novedosas caracterizaciones de 
fallas inducidas en un motor instrumentado con un acelerómetro mediante el método probabilístico NaiveBayes, el cual demostró que detecta y clasifica con precisión fallas mecánicas que ocurren en múltiples componentes simultáneamente [5]. En este contexto también los investigadores han trabajado sobre la clasificación de fallas en MCI utilizando indicadores calculados con técnicas en el dominio de tiempo y algoritmos de selección de variables, basado en análisis de varianza [6]. Aunque, ya existen avances en el desarrollo de estrategias de monitoreo y diagnóstico aplicadas a MCI, aún se tiene la necesidad de continuar con el desarrollo de nuevas estrategias que ayuden en la identificación de fallos que pueden aparecer de forma repentina.

Por lo tanto, la contribución de este trabajo se basa en la propuesta y desarrollo de una metodología de monitoreo de condición, aplicada al diagnóstico y detección de fallas producidas en el sistema de encendido de MCI mediante el análisis de señales de vibración. Las novedades de la metodología propuesta incluyen: a) la adquisición de señales de vibración para describir la operación continua de trabajo de un MCI; b) la caracterización de señales de vibración mediante un conjunto de indicadores estadísticos en el dominio del tiempo para obtener los patrones representativos de la condición de operación del MCI y c) la consideración de técnicas de reducción de dimensionalidad para fusionar la información de los diferentes indicadores estadísticos y obtener una representación visual de las condiciones evaluadas. La validación de la metodología propuesta se realiza mediante el análisis de señales de vibración adquiridas experimentalmente en un MCI que opera bajo condiciones normales y condiciones anormales producidas por fallas en el sistema de encendido.

\section{FUNDAMENTACIÓN TEÓRICA}

\section{A. Técnicas para el cálculo de indicadores}

El objetivo de las técnicas de procesamiento de señales es el de caracterizar una señal específica para resaltar y obtener sus mejores atribuciones. La mayoría de técnicas de procesamiento incluyen análisis en el dominio del tiempo, de la frecuencia y tiempo-frecuencia. Referente a las técnicas del dominio del tiempo, el cálculo de indicadores estadísticos sigue prevaleciendo como una de las mejores estrategias para modelar tendencias y cambios de señales, incluso, el cálculo de indicadores estadísticos es preferido debido al bajo costo computacional requerido [7]. Por otra parte, las técnicas del dominio de la frecuencia y tiempo-frecuencia, pueden ser de mayor complejidad y requieren el previo conocimiento de su uso para obtener buenas representaciones y resultados de alto rendimiento; dentro de estas técnicas destacan la transformada rápida de Fourier (FFT, Fast Fourier Transform), la transformada corta de Fourier (STFT, Short-time Fourier Transform) y transformadas Wavelet (CWT, Continuous Wavelet Transform) [8], [9], [10].

\section{B. Técnicas de reducción de indicadores}

Es posible utilizar una gran cantidad de indicadores pensando en poder aumentar las capacidades de discriminación entre fallas, sin embargo, este aumento de indicadores no garantiza el incremento de información importante y relacionada con las diferentes condiciones de operación. Por esta razón, se requiere utilizar algunas de las diferentes estrategias y procedimientos de reducción de indicadores [11]. El uso de técnicas de reducción de indicadores aplicadas en estrategias de monitoreo de condición y diagnóstico de fallas han demostrado ser de gran utilidad para resaltar las diferencias que existen entre distintos patrones de falla y para la reducción de la dimensionalidad de un conjunto de datos representados en un espacio dimensional d. Dentro de estas técnicas, las más usadas y conocidas son: Análisis de Componentes Principales (PCA, Principal Component Analysis) y Análisis Discriminante Lineal (LDA, Linear Discriminant Analysis) [12].

La PCA tiene sus bases en el álgebra lineal y es una técnica no supervisada por no requerir información de la clase a la que pertenecen las muestras analizadas. Su enfoque de reducción de la dimensionalidad se basa en mantener la variabilidad del conjunto de datos originales. En forma más específica, cuando se aplica la técnica PCA sobre un conjunto de indicadores, se extrae un nuevo conjunto de indicadores llamados componentes principales y cada uno de estos es una combinación lineal de los indicadores originales. En adición a esto, en las componentes principales no hay información repetida por ser ortogonales entre sí; de esta manera se mantiene la variabilidad del conjunto de indicadores originales [13]. El proceso matemático es bastante simple 
utilizando el criterio del error cuadrado para minimizar la distancia entre el vector $x_{w}$ y los demás vectores, como en la ecuación (1).

$$
J_{0}\left(x_{0}\right)=\sum_{i=1}^{N}\left\|x_{w}-x_{i}\right\|^{2}
$$

Primero, se calcula la media del vector dimensional $d$ y la matriz de covarianza $\mu$, para el todo el conjunto de datos normalizados. A continuación, se calculan los vectores propios $x_{\lambda-i}$ y los valores propios $\lambda_{i}$ de la matriz de covarianza y se ordenan de acuerdo con el valor propio decreciente, finalmente, se eligen los $k$ vectores propios más significativos para realizar la transformación y proyección de los nuevos indicadores, véase la ecuación (2).

$$
Y_{i}=x_{\lambda-i} * X_{i}
$$

Por otro lado, la LDA es una técnica supervisada de reducción de la dimensionalidad cuyo objetivo se centra en maximizar tanto como sea posible la separación lineal entre conjuntos de muestras que pertenezcan a diferentes clases o diferentes condiciones. Para casos prácticos de aplicación en la identificación de fallas, la LDA es una técnica apropiada que puede conducir a la identificación de diferentes condiciones. considerando que esta técnica engloba problemas de múltiples clases con $C$ clases compuestas por $N$ muestras, la LDA calcula una matriz de dispersión entre clases con la ecuación (3).

$$
S_{b}=\sum_{j=1}^{C} N_{j}\left(m_{j}-\bar{m}\right)\left(m_{j}-\bar{m}\right)^{T}
$$

donde $N_{j}$ corresponde al número total de muestras de la j-ésima clase $C_{j}$, teniendo en cuenta todas las clases $\bar{m}$ es la media de todas las muestras y $m_{j}$ es la media de la clase $C_{j}$. También considera el cálculo de la matriz de dispersión dentro de las clases como en la ecuación (4). El vector óptimo de proyección $W_{L D A}$ que es elegido por la LDA permite obtener clases bien separadas con la ecuación (5).

$$
\begin{aligned}
S_{w} & =\sum_{j=1}^{C} \sum_{i=1}^{N_{j}}\left(x_{i}^{j}-m_{j}\right)\left(x_{i}^{j}-m_{j}\right)^{T}=\sum_{j=1}^{C} S_{w_{j}} \\
W_{L D A} & =\arg \max \frac{\left|W^{T} S_{b} W\right|}{\left|W^{T} S_{w} W\right|}=\left[\begin{array}{llll}
w_{1} & w_{2} & \cdots & w_{m}
\end{array}\right]
\end{aligned}
$$

donde $\left\{w_{i} \mid i=1,2, \cdots, m\right\}$ pertenece al conjunto de vectores propios generalizados también conocidos como vectores discriminantes de la matriz $S_{b}$ y $S_{w}$ que corresponden a los valores propios generalizados de mayor relevancia de la clase $C-1\left\{\lambda_{i} \mid i=1,2, \cdots, m\right\}$. De este modo, la extracción de indicadores que resulta en $V$ se obtiene a través de la proyección del conjunto de datos original de indicadores $X$ en el espacio de dimensión reducida $W_{L D A}$ como se ve en la ecuación (6) [11]. El desarrollo teórico-matemático de ambas técnicas es bien conocido y sus detalles pueden consultarse en [12], [14],[15].

$$
V=W_{L D A}{ }^{T} X
$$




\section{METODOLOGÍA}

\section{A. Experimento}

La metodología propuesta es aplicada sobre datos experimentales adquiridos de un MCI, la puesta en marcha del experimento consistió en instalar un acelerómetro triaxial de bajo costo (ADXL345) sobre una parte solida del motor, específicamente fue instalado sobre el bloque del MCI. Para la adquisición de señales se desarrolló un sistema de adquisición de datos bajo el lenguaje de programación en hardware basado en tecnología FPGA (Field Programmable Gate Array). El sistema de adquisición de datos cuenta con su etapa de acondicionamiento correspondiente para evitar el aliasing. Las señales de vibración se adquirieron experimentalmente bajo dos condiciones diferentes de operación del MCI, es decir, bajo condición sana o normal y bajo condición de falla en el sistema de encendido. Para las dos condiciones evaluadas en el MCI, las señales de vibración fueron adquiridas durante 60 segundos cuando el MCI se encontraba operando en modo ralentí aproximadamente a $950 \mathrm{rpm}$.

La condición de falla en el MCI se indujo de forma artificial mediante la desconexión de uno de los cables de bujías, lo cual produjo que la bujía de uno de los cilindros dejara de producir chispa y así afectar a la combustión del MCI. El acelerómetro fue operado con una frecuencia de muestreo de $3200 \mathrm{~Hz}$ y los datos adquiridos fueron almacenados en una PC para su posterior análisis.

\section{B. Metodología de diagnóstico propuesta}

La metodología de diagnóstico propuesta para la detección de fallas ocasionadas en el sistema de ignición de un MCI consta principalmente de cuatro etapas diferentes como se muestra en el diagrama a bloques de la Fig. 1.

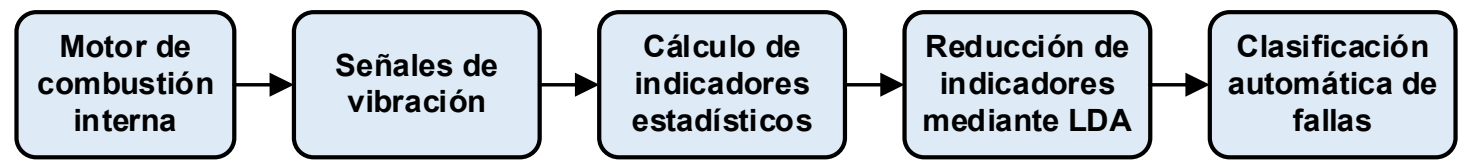

Fig. 1. Diagrama bloques la metodología de diagnóstico propuesta para la identificación de fallas en motores de combustión internar mediante el análisis de señales de vibración.

Primero: se realiza el monitoreo continuo de la operación sobre el MCI, dicho monitoreo considera la adquisición de señales de vibración que son adquiridas mediante un sistema de adquisición de datos de arquitectura abierta y enviadas para ser almacenados en una computadora personal para su análisis posterior. Cabe mencionar que los datos adquiridos corresponden a la medición de vibraciones producidas en el MCI que opera bajo diferentes condiciones de operación.

Segundo: después de adquirir las señales de vibración, se efectúa el cálculo de indicadores estadísticos que describirán el comportamiento y tendencias las señales de vibración adquiridas; así, se realiza la caracterización de las señales adquiridas mediante la estimación numérica de dicho conjunto de cuatro indicadores estadísticos del dominio de tiempo. El objetivo principal es obtener la caracterización de los patrones de las señales de vibración que representan diferentes condiciones de operación del MCI.

Tercero: en la etapa de reducción de indicadores, el conjunto original de cuatro indicadores estadísticos es sometidos a un proceso de compresión y transformación de base mediante la aplicación de las técnicas de reducción de la dimensionalidad PCA y LDA. La finalidad de la aplicación del proceso de reducción es obtener una representación visual en un espacio bidimensional $(2 D)$; además, el uso de estas técnicas permite fusionar la información de cada uno de los indicadores estadísticos lo que conduce a una mejor caracterización de las condiciones evaluadas.

Cuarto: mediante la aplicación de un clasificador basado en una red neuronal de estructura simple se obtiene el diagnóstico y clasificación automática de la aparición de fallas en el MCI. El uso de la red neuronal como herramienta de clasificación ayuda a generar una estructura de diagnóstico automática debido que las salidas del diagnóstico las basa en el análisis de los resultados obtenidos por el uso de las técnicas 
PCA y LDA. Es decir, dicho clasificador se enfocará en realizar la clasificación basándose en el análisis de las proyecciones resultandos en el espacio bidimensional $(2 D)$.

\section{RESULTADOS Y DISCUSIONES}

El procesamiento de las señales adquiridas y la aplicación de la metodología de diagnóstico propuesta se hizo bajo el entorno de programación de Matlab ${ }^{\circledR}$. Como anteriormente se mencionó, la adquisición de las señales de vibración se realizó durante 60 segundos cuando el MCI operaba a aproximadamente $950 \mathrm{rpm}$. En la Fig. 2 se muestran las señales de vibración adquiridas para ambas condiciones de operación, sano y falla, respectivamente, se puede observar también un claro incremento en la amplitud de las vibraciones producidas por el MCI cuando se encuentra operando bajo la influencia del fallo inducido en el sistema de encendido, este incremento de vibraciones tiene una buena representación por estar asociado al mal funcionamiento del MCI y tendrá un patrón específico representativo.
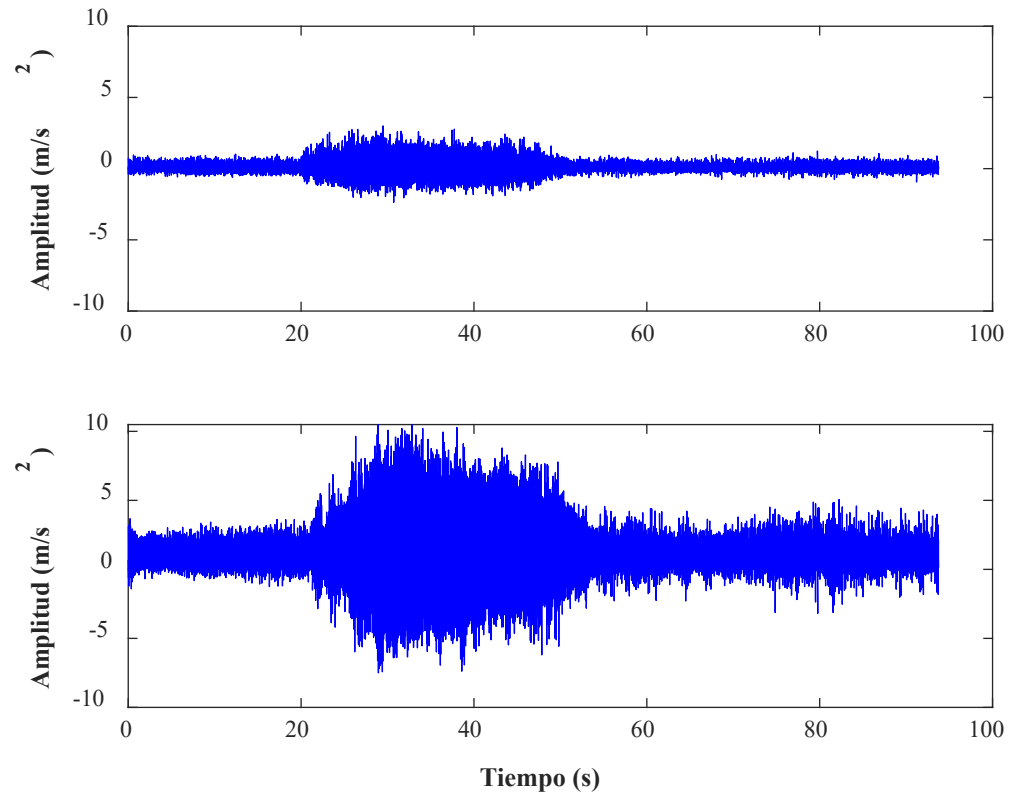

Fig. 2. Señales de vibración adquiridas durante la operación del $\mathrm{MCl}$ en operaciones de trabajo sana (superior) y una condición de falla ocasionada en el sistema de ignición (inferior).

Posteriormente, cada una de las señales de vibración adquiridas fue segmentada en partes iguales de 0.5 segundos con la finalidad de formar un conjunto de muestras consecutivas. Enseguida, para obtener una caracterización de las señales adquiridas, sobre cada uno de los segmentos de las señales se calcularon los cuatro indicadores estadísticos en el dominio del tiempo: valor eficaz (RMS-Root Mean Square), ecuación (7), la desviación estándar $(\sigma)$, de acuerdo a la ecuación (8), el sesgo $\left(S_{k}\right)$, con la ecuación (9), y la curtosis $\left(K_{u r}\right)$, como se muestra la ecuación (10) [11], [16].

$$
R M S=\sqrt{\frac{\sum_{i=i}^{n}\left(x_{i}\right)^{2}}{n}}
$$




$$
\begin{gathered}
\sigma=\sqrt{\frac{\sum_{i=1}^{n}\left(x_{i}-\bar{x}\right)^{2}}{n}} \\
S_{k}=\frac{\sum_{i=1}^{n}\left(x_{i}-\bar{X}\right)^{3}}{\sigma^{3}} \\
K_{u r}=\frac{\frac{1}{n} \sum_{i=1}^{n}\left(x_{i}-\bar{X}\right)^{4}}{\sigma^{4}}
\end{gathered}
$$

Sucesivamente, con el objetivo de obtener una representación visual de las condiciones de operación evaluadas en el MCI (sano y falla), el conjunto de indicadores estadísticos estimados fue sometido al proceso de reducción y transformación de base mediante las técnicas PCA y LDA. Como resultado de este proceso, se obtuvieron las gráficas mostradas en la Fig. 3 y la Fig. 4, respectivamente.

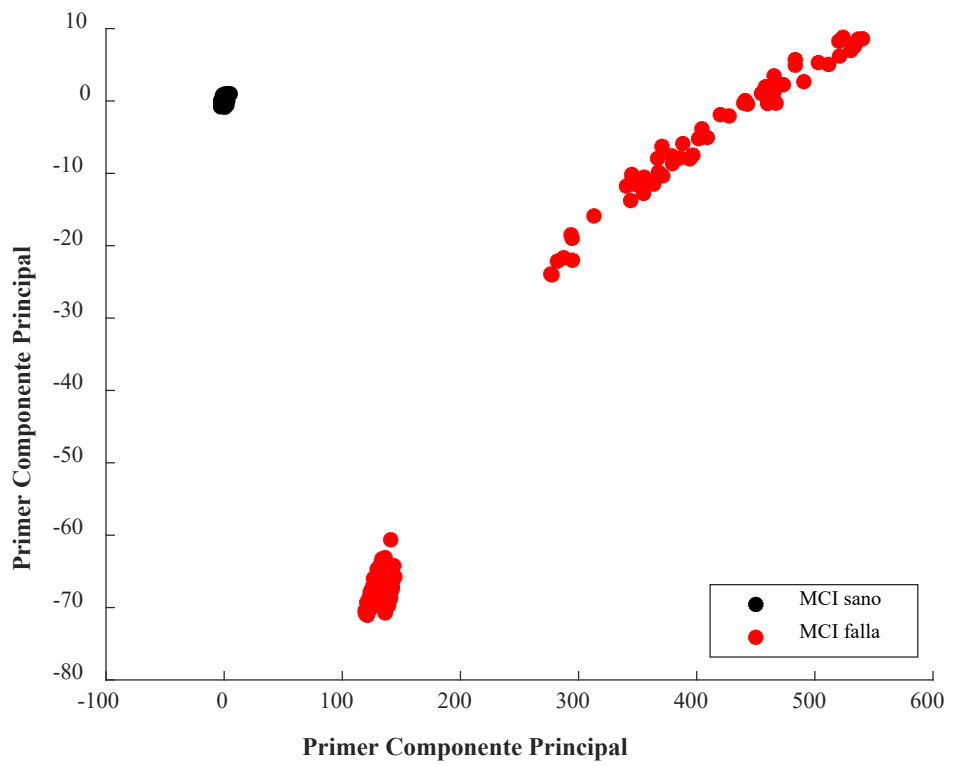

Fig. 3. Proyección resultante obtenida tras aplicar la PCA al conjunto original de indicadores estadísticos calculados de las señales de vibración disponibles. 


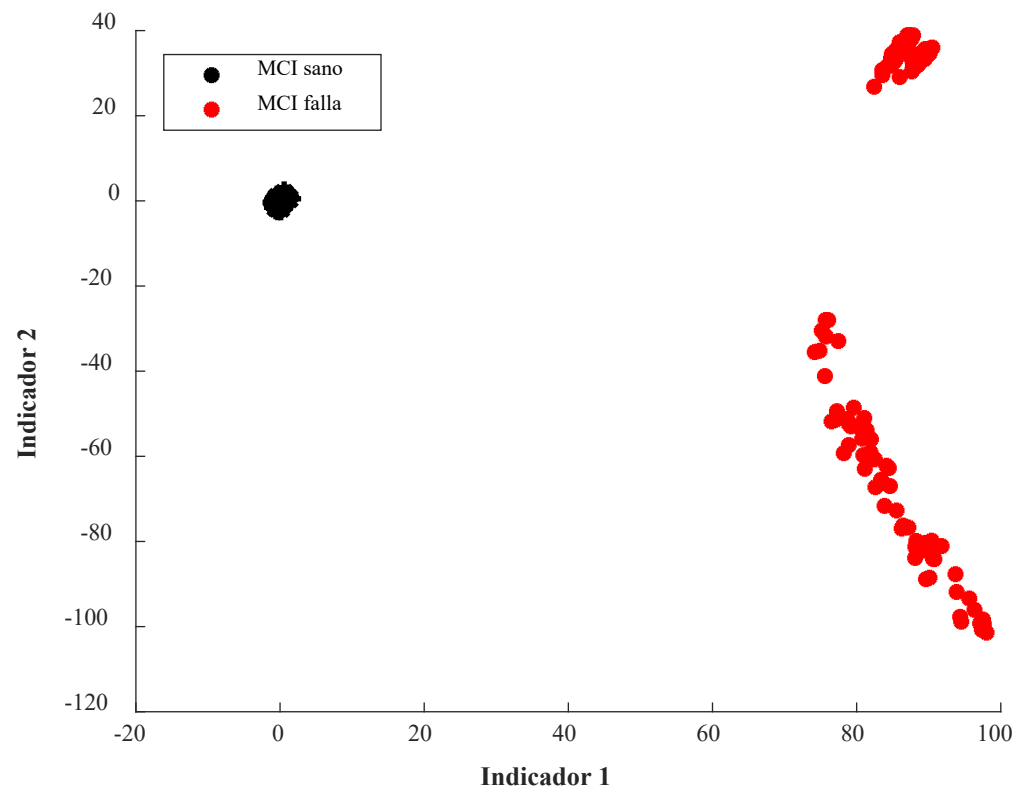

Fig. 4. Proyección resultante al aplicar la LDA como técnica de reducción de indicadores sobre el conjunto de indicadores estadísticos que caracterizan las señales de vibración cuando el $\mathrm{MCl}$ opera bajo condiciones diferentes de operación.

De los resultados obtenidos al aplicar la reducción de indicadores debe mencionarse que la aplicación de la PCA para las diferentes condiciones, sano y falla evaluados a dos velocidades de giro del MCI, 950 rpm y 5000 rpm; lo que muestra que se mantuvo la variabilidad del conjunto de señales de vibración de MCI como se muestra en la Fig. 3, y la separación de condiciones se obtiene debido a que existe una desviación en la varianza de los indicadores de la condición evaluadas. Por otra parte, al aplicar la LDA se obtiene una maximización de la separación entre clases y una minimización de la distancia entre datos de cada condición como se muestra en la Fig. 4. Estos resultados prueban que las técnicas de reducción de indicadores son una herramienta útil que pueden conducir a la detección de fallas en un MCI, incluso, el uso de estas técnicas también lleva a obtener una representación visual para proyectar un conjunto original de datos en 2D.

Para validar la aplicación del método propuesto, la misma condición de falla en el MCI fue evaluada a una velocidad de giro variable, por lo tanto, un conjunto de señales de vibración fue también adquirido cuando el MCI operaba de aproximadamente $900 \mathrm{rpm}$ a $5000 \mathrm{rpm}$; es decir, se adquirieron durante un periodo de incremento de velocidad del motor para observar el comportamiento de la falla. Posteriormente, la metodología de diagnóstico fue aplicada como se explicó anteriormente y el conjunto de muestras correspondientes a esta operación de fallo fue proyectado sobre las representaciones visuales obtenidas tras aplicar la PCA y LDA respectivamente. Los resultados de la evaluación del fallo en progreso (evolución) en un rango de velocidad de giro del motor de $900 \mathrm{rpm}$ a $5000 \mathrm{rpm}$, y la reducción de la dimensionalidad se muestran en las Fig. 5 y Fig. 6 respectivamente. Así, como se puede observar en las Fig. 5 y Fig. 6, la metodología de diagnóstico y detección de falla en el sistema de encendido de un MCI también es capaz de determinar que existe un área en las proyecciones resultantes en donde prevalece el comportamiento y se muestra la evolución de la aparición del fallo en el sistema de ignición del MCI.

De acuerdo con los resultados obtenidos, la metodología propuesta de diagnóstico y detección de fallas en MCI permite detectar de forma precisa la aparición de fallas en el sistema de encendido, independientemente de la velocidad de giro del MCI. Además, en el caso de aplicación de la técnica LDA, esta proporciona mejores resultados debido a que se trata de una técnica enfocada a la solución de problemas de múltiples clases. Por otra 
parte, aunque la PCA también es capaz de separar las condiciones evaluadas, su rendimiento comenzará a decrementar en cuanto se incremente el número de condiciones.

o

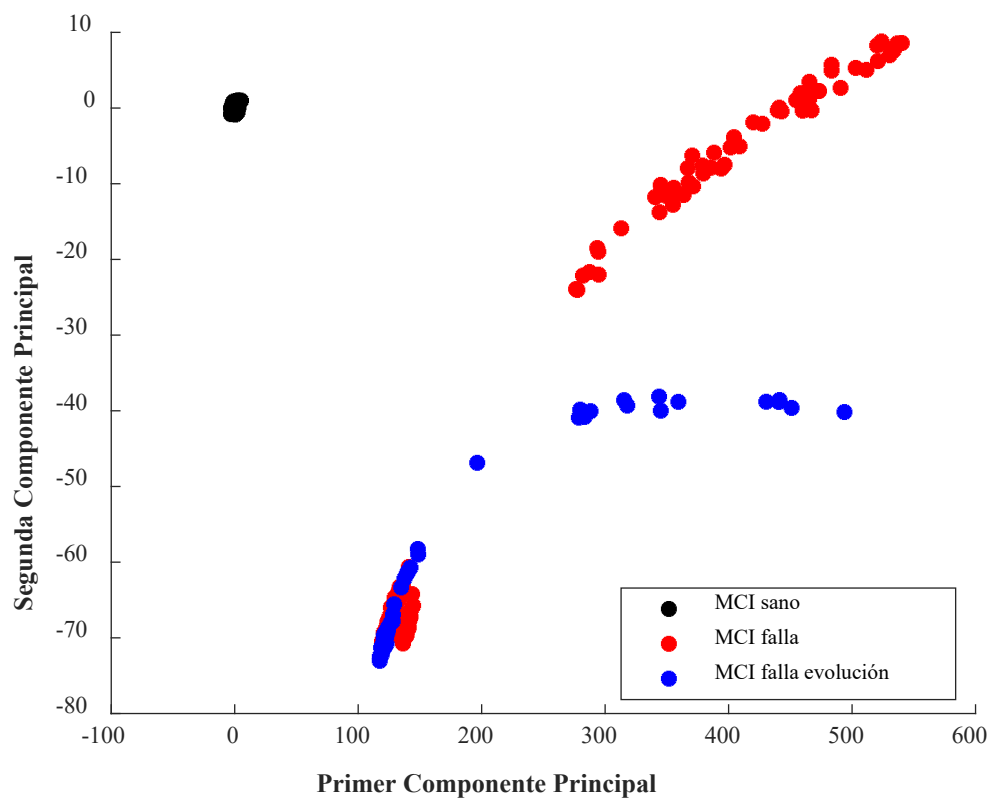

Fig. 5. Proyección resultante que se obtuvo al aplicar la PCA al conjunto original de indicadores que muestra el comportamiento de las muestras que corresponden a la evaluación del fallo en un rango de revoluciones entre $900 \mathrm{rpm}$ a $5000 \mathrm{rpm}$.

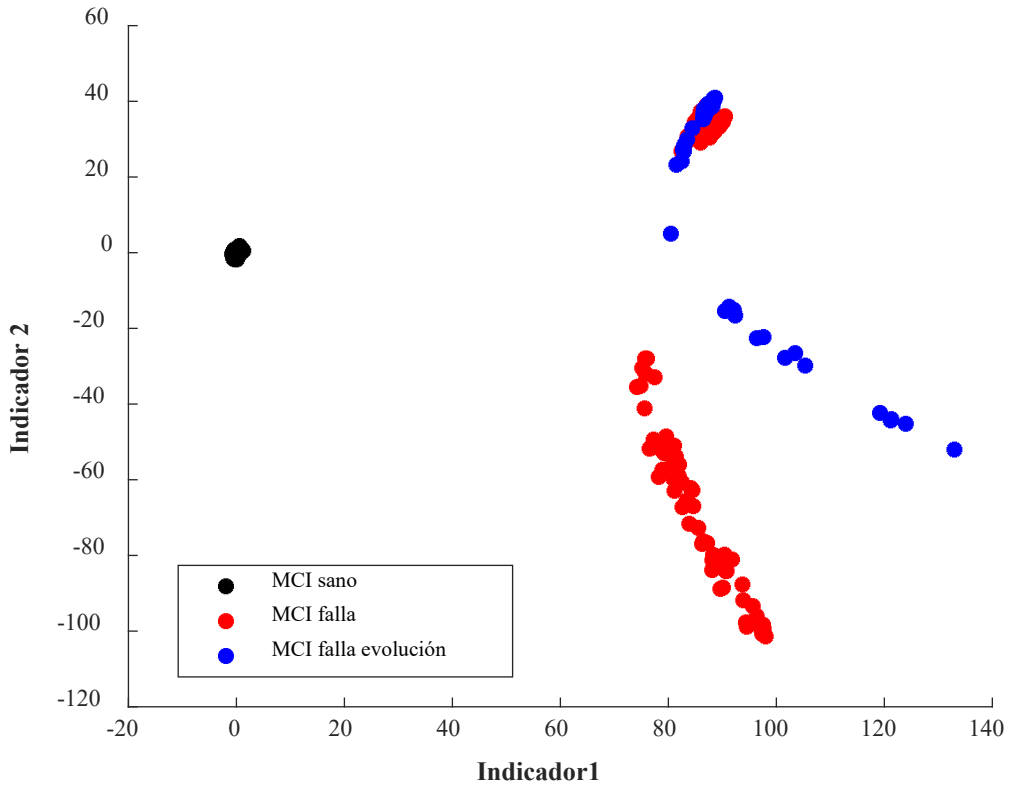

Fig. 6. Proyección resultante obtenida al aplicar la LDA como técnica de reducción de indicadores donde se muestra el comportamiento de un conjunto de muestras que corresponden a la evaluación del fallo en un rango de revoluciones entre 900 rpm a $5000 \mathrm{rpm}$. 
Respecto a la aplicación del clasificador de fallas automático, se utilizó una estructura básica de una red neuronal de tres capas, en la capa de entrada la red neuronal consta de dos neuronas que corresponden al número de la dimensionalidad a la cual fue reducido el conjunto original de indicadores estadísticos; es decir, en la capa de entrada se evaluar las muestras proyectadas en esos espacios reducidos de dos dimensiones. En la capa oculta la red neuronal se conformó con diez neuronas como en algunas de las aplicaciones de clasificación lo recomienda; finalmente, en la capa de salida la red neuronal conto con dos neuronas debido a que dos condiciones son las que fueron evaluadas, condición sana y de falla, respectivamente. Por lo tanto, cuando fueron evaluados los conjuntos de muestras obtenidos en las proyecciones obtenidas tras aplicar la PCA y LDA, la red neuronal obtuvo porcentajes de clasificación global promedio del $99.8 \%$ y $100 \%$ durante el entrenamiento y evaluación de dicha red neuronal. La red neuronal es una red de propagación hacia atrás y fue entrenada durante 50 épocas usando funciones de activación sigmoidal.

\section{CONCLUSIONES}

El desarrollo de esta metodología de diagnóstico incluye el uso de técnicas de reducción de indicadores para la aplicación del diagnóstico de falla de encendido por chispa en un $\mathrm{MCI}$, probando que la reducción de indicadores estadísticos mediante la PCA y la LDA permiten determinar la aparición de fallas a diferentes velocidades de operación del MCI. Así, mientras que PCA mantiene la variabilidad de las señales, la LDA maximiza la separación entre clases, sano y fallo. Además, se demostró que el uso de señales de vibración y su caracterización mediante el cálculo de indicadores estadísticos son herramientas fiables para ser aplicadas con fines de monitoreo y diagnóstico en MCI. Además, el uso e implementación de herramientas de inteligencia artificial, como redes neuronales, ayuda a generar respuestas automáticas en durante la tarea de monitoreo y diagnóstico que pueden ser aplicadas en MCI. Una de las prospectivas de este trabajo reside en la evaluación de otras fallas en el MCI para generar una metodología de monitoreo y diagnóstico con mayores capacidades de detección, incluso, la implementación de esta metodología en hardware podría ayudar a generar herramientas de diagnóstico en tiempo real.

\section{REFERENCIAS}

[1] L. Warren y J. H. Haynes, Chevrolet/GMC Camionetas. Manual de Reparación, vol. 99040. California, USA: Haynes de Norte América, 1995.

[2] A. Moosavian, G. Najafi, B. Ghobadian, M. Mirsalim, S. M. Jafari, y P. Sharghi, "Piston scuffing fault and its identification in an IC engine by vibration analysis", Appl. Acoust., vol. 102, pp. 40-48, 2016, doi: https://doi.org/10.1016/j.apacoust.2015.09.002

[3] J. Da Wu y C. Q. Chuang, "Fault diagnosis of internal combustion engines using visual dot patterns of acoustic and vibration signals", NDT E Int., vol. 38, núm. 8, pp. 605-614, 2005, doi: https://doi.org/10.1016/j.ndteint.2005.02.007

[4] S. Delvecchio, P. Bonfiglio, y F. Pompoli, "Vibro-acoustic condition monitoring of Internal Combustion Engines: A critical review of existing techniques", Mechanical Systems and Signal Processing, vol. 99. Academic Press, pp. 661-683, 15-ene-2018, doi: https://doi.org/10.1016/j.ymssp.2017.06.033

[5] J. Flett y G. M. Bone, "Fault detection and diagnosis of diesel engine valve trains", Mech. Syst. Signal Process., vol. 72-73, pp. 316-327, 2016, doi: https://doi.org/10.1016/j.ymssp.2015.10.024

[6] E. Ftoutou, M. Chouchane, y N. Besbès, "Internal combustion engine valve clearance fault classification using multivariate analysis of variance and discriminant analysis", Trans. Inst. Meas. Control, vol. 34, núm. 5, pp. 566577, 2012, doi: https://doi.org/10.1177/0142331211408492

[7] J. J. Saucedo-Dorantes, M. Delgado-Prieto, R. A. Osornio-Rios, y R. De Jesus Romero-Troncoso, "Multifault Diagnosis Method Applied to an Electric Machine Based on High-Dimensional Feature Reduction", IEEE Trans. Ind. Appl., vol. 53, núm. 3, pp. 3086-3097, 2017, doi: https://doi.org/10.1109/TIA.2016.2637307

[8] K. Jafarian, M. Mobin, R. Jafari-Marandi, y E. Rabiei, "Misfire and valve clearance faults detection in the combustion engines based on a multi-sensor vibration signal monitoring”, Meas. J. Int. Meas. Confed., vol. 128, núm. March, pp. 527-536, 2018, doi: https://doi.org/10.1016/j.measurement.2018.04.062

[9] A. Taghizadeh-Alisaraei y A. Mahdavian, "Fault detection of injectors in diesel engines using vibration timefrequency analysis", Appl. Acoust., vol. 143, pp. 48-58, 2019, doi: https://doi.org/10.1016/j.apacoust.2018.09.002 
[10] J. Da Wu y J. C. Chen, "Continuous wavelet transform technique for fault signal diagnosis of internal combustion engines”, NDT E Int., vol. 39, núm. 4, pp. 304-311, jun. 2006, doi: https://doi.org/10.1016/j.ndteint.2005.09.002

[11] J. J. Saucedo-Dorantes, "Metodologías para mejorar la confiabilidad del diagnóstico de fallas en cadenas cinemáticas basado en algoritmos inteligentes y fusión de datos", tesis de doctorado, mecatrónica, Universidad Autónoma de Querétaro, San Juan del Río, Querétaro, México, 2017.

[12] R. O. Duda, P. E. Hart, y D. G. Stork, Pattern Classification, Second Edi. New York, N.Y., U.S.A: John Wiley \& Sons, 2012

[13] X. Wan, D. Wang, P. W. Tse, G. Xu, y Q. Zhang, “A critical study of different dimensionality reduction methods for gear crack degradation assessment under different operating conditions", Meas. J. Int. Meas. Confed., vol. 78, pp. 138-150, 2016, doi: https://doi.org/10.1016/j.measurement.2015.09.032

[14] S. Theodoridis y K. Koutroumbas, Pattern Recognition, Third Edit. San Diego, California, USA: Academic Press, 2006.

[15] S. I. Grossman y J.-J. Flores-Godoy, Álgebra Lineal, Septima Ed. D.F., México: McGraw-Hill, 2012.

[16] A. Rustom J., Estadistica Descriptiva, Probabilidad e Inferencia, Primera ed. Santiago, Chile: Universidad de Chile, 2012. 